\title{
Purification and Characterization of the Amino-Terminal Domain of Lumazine Protein from Photobacterium leiognathi
}

\author{
So-Young Kim, Ryu-Ryun Kim, Ji-Sun Choi, Young-Doo Kim, and Chan Yong Lee* \\ Department of Biochemistry, Chungnam National University, Daejeon 305-764, Korea. *E-mail: cylee@cnu.ac.kr \\ Received December 21, 2009, Accepted February 12, 2010
}

Key Words: Lumazine protein, Photobacterium leiognathi, Riboflavin synthase

Luminescent bacteria emit blue-green light via the correlated oxidation of long chain aldehyde and reduced riboflavin 5'phosphate $\left(\mathrm{FMNH}_{2}\right)$ catalyzed by bacterial luciferase to FMN and $\mathrm{RCOOH}$, respectively. ${ }^{1,2}$

$\mathrm{FMNH}_{2}+\mathrm{RCHO}+\mathrm{O}_{2} \rightarrow \mathrm{FMN}+\mathrm{H}_{2} \mathrm{O}+\mathrm{RCOOH}+$ light

Lumazine protein was first isolated from the luminescent

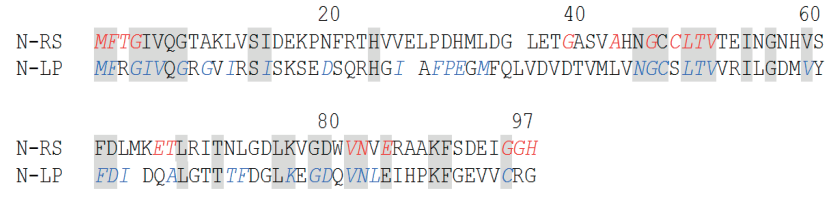

(B)

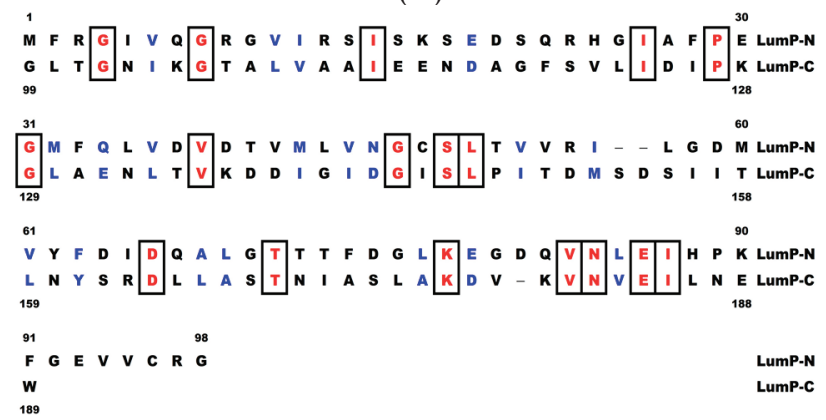

Figure 1. (A) Amino acid sequence similarity between N-terminal parts of riboflavin synthase from E. coli (N-RS) and lumazine protein from P. leiognathi (N-LP). Amino acid residues that are highly conserved among riboflavin synthases or lumazine proteins are in italicized. Identical positions are shadowed. (B) Amino acid sequence similarity between $\mathrm{N}$ - and $\mathrm{C}$-terminal parts of lumazine protein from $P$. leiognathi. Identical residues are boxed. The gene coding for the polypeptides of N-terminal of LumP was synthesized by PCR as shown in Table 1. marine bacterium, Photobacterium Phosphoreum. ${ }^{3}$ The intense fluorescence of the bacterial protein is caused by non-covalently bound 6,7-dimethyl-8-ribityllumazine, ${ }^{4,5}$ a compound that is also known to serve as the direct biosynthetic precursor of riboflavin (vitamin $\mathrm{B}_{2}$ ). ${ }^{6,7}$ The participation of lumazine protein in bacterial bioluminescence is believed to shift the emission maximum and increase the quantum yield of bacterial luminescence. ${ }^{4,5}$ More specifically, lumazine protein is assumed to become optically excited by radiationless transition. ${ }^{8,9}$

Lumazine protein is a member of the riboflavin synthase superfamily. ${ }^{10}$ Amino acid comparisons have shown close similarity (Figure 1A) between lumazine protein (LumP) and riboflavin synthase (RS) catalyzing the conversion of 6,7-dimethyl8-ribityllumazine into riboflavin. It is interesting to note that the lux operon of Photobacterium species is flanked by the related genes for lumazine protein (LumP) and for riboflavin synthase (RS), and transcribed in opposite direction. ${ }^{11}$

The internal amino acid sequence similarity between N-terminal half and C-terminal half of the lumazine protein (Figure 1B) as well as the X-ray crystal structure of lumazine protein ${ }^{12}$ suggest that lumazine protein folds into two domains with closely similar folding topology. The comparison with the paralogous of riboflavin synthase (RS) whose three-dimensional structure of $\mathrm{N}$-terminal domain has been determined by $\mathrm{NMR}^{13}$ and X-ray crystallization $^{14,15}$ also supports the folding topology of lumazine protein. In this study, therefore, a recombinant gene specifying the N-terminal domain of the lumazine protein (N-LumP) was constructed and the cognate protein was purified. In addition, taken together N-terminal domain of riboflavin synthase (N-RS), quaternary structure and fluorescence characteristic of N-LumP were examined.

The gene coding for amino-terminal domain of lumazine protein (pPHL36-N), was produced by Polymerase Chain Reaction (PCR) with Vent DNA polymerase (New England Biolabs). Plasmid pPHL $36^{16}$ containing the whole gene for luma-

Table 1. Oligonucleotides used for the construction of the N-terminal domain of lumazine protein (N-LumP). Restriction sites are underlined and the translated amino-acid sequences are shown under the nucleotide sequences. The whole amino acid sequences of N-LumP (MFRGIV

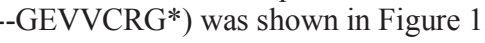

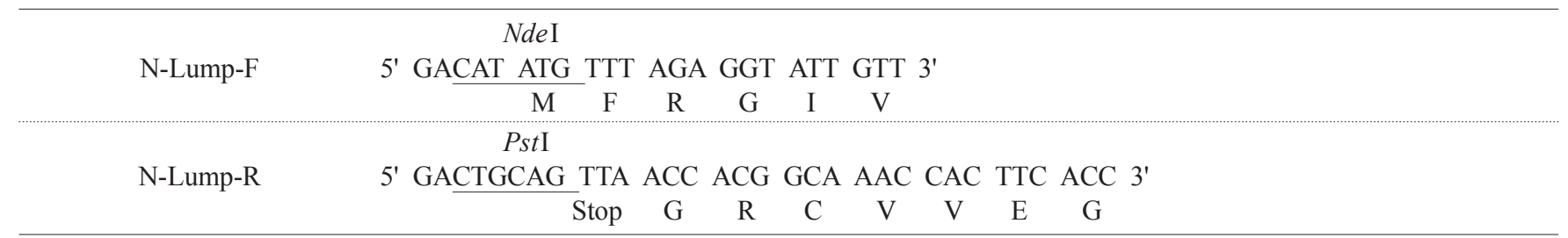


(A)

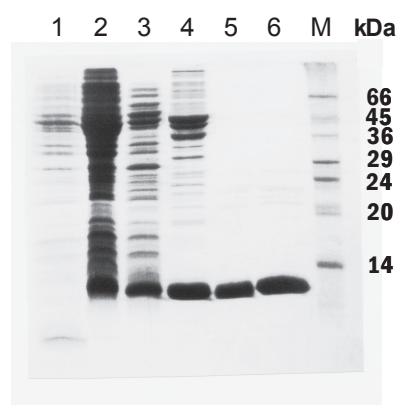

(B)

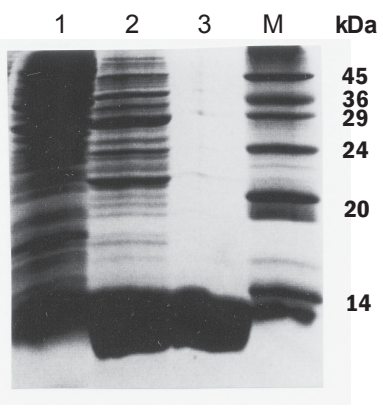

Figure 2. SDS-PAGE during the purification steps of protein. (A) Purification of amino- terminal domain of riboflavin synthase (N-RS) from E. coli. lane 1, uninduced; lane 2, induced crude extract; lane 3, supernatant of crude extract after sonication and centrifugation; lane 4, after purification of N-RS by Sepharose; lane 5, N-RS after Superdex; lane 6, after ultrafiltration; lane M, protein size markers. (B) Purification of amino-terminal domain of lumazine protein (N-LumP). Lane 1 , extract of cells containing the plasmid pPHL36-N for the gene coding for the N-LumP from P. leiognathi; lane 2, after purification of N-LumP by Sepharose; lane 3, N-LumP after Superdex; lane M, protein size markers.

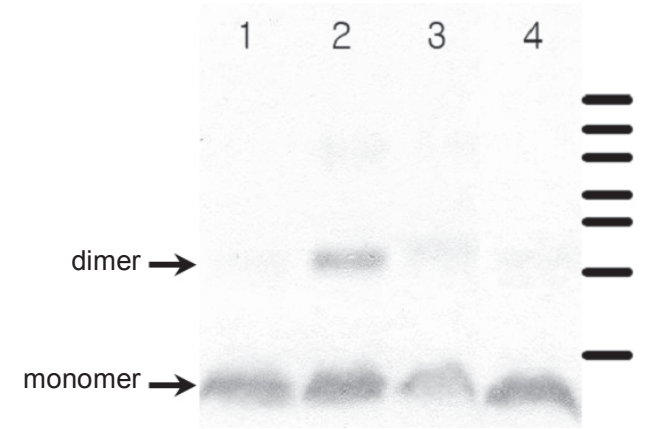

Figure 3. Cross-linking of N-RS and N-LumP with glutaraldehyde. Lane 1, N-RS without glutaraldehyde; lane 2, N-RS with glutaraldehyde; lane 3, N-LumP without glutaraldehyde; lane 4, N-LumP with glutaraldehyde. The positions of migration of protein standards are marked on right $(14,20,24,29,36,45,66 \mathrm{kDa}$, respectively).

zine protein was used as template and the two flanking primers (N-LumP-F and N-LumP-R) are shown in Table 1. The constructed recombinant plasmid (pPHL36-N) was sequenced by the automated Sanger's dideoxy-termination method. The translated amino acid sequences of the protein coded by N-LumP gene, produced by PCR, is shown in Figure 1A (N-LP) and 1B (upper amino acid sequences). The amplified DNA was purified by agarose gel electrophoresis and the DNA was digested with NdeI and PstI. The resulting fragment was purified using the QIAquick PCR purification kit and ligated into the plasmid pT7-7 that had been digested with the same restriction enzymes. The ligation mixtures were transformed into E. coli XL-1 blue cells.

A recombinant $E$. coli strain carrying the plasmid pPHL36-N produced N-LumP to a level of $5 \sim 10 \%$ of cell protein following induction with IPTG. The apparent mass as observed by SDS/PAGE was about $11 \mathrm{kDa}$ in agreement with the predicted

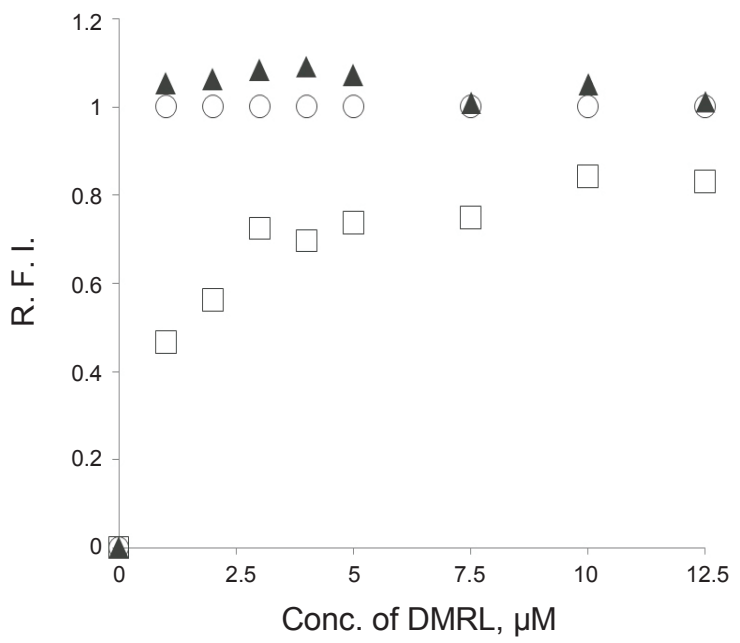

Figure 4. Relative fluorescence intensity with increasing concentrations of fluorescent ligand 6,7-dimethyl-8-ribityllumazine. Relative fluorescence intensity (R.F.I.) were calculated by normalization of fluorescence intensity of 6,7-dimethyl-8-ribityllumazine (DMRL) itself. excitation, $410 \mathrm{~nm}$; emission, $490 \mathrm{~nm}$. ○, DMRL; $\mathbf{\Delta}$, DMRL with $5 \mu \mathrm{M}$ of N-LumP; $\square$, DMRL with $5 \mu \mathrm{M}$ of N-RS. The ligand-free N-RS and the N-LumP, do not show any fluorescence.

mass of the recombinant protein of 10,550 Da (Figure 2). Recombinant N-LumP and N-RS were purified by anion exchange chromatography as described in the Experimental Section (Figure 2). It was shown that the N-RS was eluted earlier than $\mathrm{N}$-LumP on gel filtration. To estimate the size of these proteins in the native state, ribonuclease and chymotrypsinogen were also loaded as standard proteins, and elution volumes were determined by monitoring the absorption of the eluate at $280 \mathrm{~nm}$. The N-LumP was eluted from a Superdex 75 column at $11 \mathrm{kDa}$ close to ribonuclease $(13.7 \mathrm{kDa})$ whereas $\mathrm{N}-\mathrm{RS}$ was eluted at $22 \mathrm{kDa}$ close to chymotrypsinogen $(25.0 \mathrm{kDa})$.

To further investigate the quaternary structures of N-RS and $\mathrm{N}$-LumP, cross-linking was performed with glutaraldehyde (Figure 4). As shown in Figure 3, in addition to monomeric species $(11 \mathrm{kDa}), \mathrm{N}-\mathrm{RS}$ remarkably forms the high molecular weight band $(22 \mathrm{kDa})$ corresponding to dimeric structure in presence of $0.05 \%$ glutaraldehyde (lane 2 ). In contrast, N-LumP was mainly present as a momeric protein in absence (lane 3 ) or in presence of cross-linker (lane 4). These results supported that the quaternary structures of the two proteins are different, i.e., the native $\mathrm{N}$-LumP is a momomeric species whereas the native $\mathrm{N}-\mathrm{RS}$ behaves as a dimer. This result is also in consistent with the previous report ${ }^{17}$ that the artificial N-RS is a $C_{2}$ symmetric homodimer.

Based on internal amino acid sequence similarity of the lumazine protein shown in Figure 1B, the monomeric protein is believed to comprise a tandem of two similar domains. In order to compare the characteristic of the proteins of N-LumP and N-RS, spectrofluorimetric titration studies were carried out. For titration experiments, the bound 6,7-dimethyl-8-ribityllumazine to the proteins after purifications was removed from dialysis against 6M urea, as described in Experimental Section.

Fluorescence titrations were performed by stepwise addition of 6,7-dimethyl-8-ribityllumazine to solution of $5 \mu \mathrm{M}$ N-RS 
and N-LumP, respectively. As shown in Figure 4, at low concentration of ligand, the fluorescence of 6,7-dimethyl-8-ribityllumazine was quenched on binding to N-RS but it remains constant on binding to N-LumP. This result indicated that the proteins of N-RS and N-LumP possess different fluorescent characteristics on binding to the fluorophore. The binding of the ligand to N-RS and N-LumP were confirmed by checking of the red shift of the maximum absorbance peak of the chromophore ligand from 410 to $414 \mathrm{~nm}$ by binding to the proteins.

According to the published data on the ligand binding of riboflavin synthase ${ }^{18,19}$ it appears that only one of the two domains can bind the fluorescent ligand. The three-dimensional structure of lumazine protein has been published recently. ${ }^{12}$ The most remarkable feature is the sequence similarity between the $\mathrm{N}$-terminal and $\mathrm{C}$-terminal domain of lumazine protein (Figure 1B). Hence, only one of the two similar domains participates directly in the photoemission, and it revealed that riboflavin is bound to a shallow cavity of the N-terminal domain of lumazine protein. ${ }^{12}$ Moreover, previous studies reported ${ }^{20}$ that replacement of amino acids in N-terminal domain had significant impact on optical properties and binding affinities whereas the mutations at $\mathrm{C}$-terminal domain did not. These results indicated that the ligand binding site is located at the N-terminal domain. Therefore, the purified protein of N-LumP will be useful and necessary for further study of the functional and spectroscopic studies as a minimal version of fluorescence domain.

Lumazine proteins are fluorescent proteins in Photobacteria that form a complex with the riboflavin precursor, 6,7-dimethyl8-ribityllumazine and they are paralogs of the enzyme, riboflavin synthase. In summary, a recombinant gene coding for the $\mathrm{N}$-terminal domain of lumazine protein from $P$. leiognath $i$ was constructed, expressed in E. coli, and the recombinant protein was purified. An artificial N-terminal domain of riboflavin synthase and the N-terminal domain of lumazine protein show different biochemical characteristics in terms of their quaternary structures of native proteins and the fluorescence properties. An artificial N-terminal domain of riboflavin synthase has been earlier shown to be dimeric, the N-terminal domain of lumazine protein is a monomer.

\section{Experimental Section}

Material and methods. Restriction enzymes were purchased from Pharmacia and New England Biolabs. T4 DNA ligase was obtained from Gibco. Primers were synthesized by MWGBiotech. Bacteria were grown on Luria Bertani (LB) medium.

Cell growing. Recombinant E. coli strains were grown in LB medium containing ampicillin $(150 \mu \mathrm{g} / \mathrm{mL})$ at $37{ }^{\circ} \mathrm{C}$ in shaking flasks to an optical density of 0.7 . Isopropyl- $\beta$-D-thiogalactopyranoside (IPTG) was added to a concentration of 0.5 $\mathrm{mM}$, and incubation was continued at $37^{\circ} \mathrm{C}$ for $4 \mathrm{~h}$. The cells were harvested by centrifugation and stored at $-70{ }^{\circ} \mathrm{C}$.

Protein purifications. Purification procedures were performed at $4{ }^{\circ} \mathrm{C}$ unless otherwise stated. Frozen cell mass $(5 \mathrm{~g})$ was thawed in $25 \mathrm{~mL}$ of $50 \mathrm{mM}$ Tris hydrochloride, $\mathrm{pH} 7.2$ containing $0.5 \mathrm{mM}$ EDTA and $0.5 \mathrm{mM}$ dithiothreitol (buffer A). The suspension was subjected to ultrasonic treatment and was then centrifuged. The supernatant was dialyzed against 10 vol- umes of buffer A and centrifuged. The supernatant was passed through a column of Q-Sepharose Fast Flow $(2 \times 18 \mathrm{~cm})$ that had been pre-equilibrated with buffer A (flow rate $1 \mathrm{~mL} / \mathrm{min}$ ). The column was washed with $100 \mathrm{~mL}$ of buffer $\mathrm{A}$ and was then developed with a linear gradient of $0 \sim 0.5 \mathrm{M} \mathrm{NaCl}$ in buffer $\mathrm{A}$. Fractions were combined, concentrated by ultrafiltration, and dialyzed against $50 \mathrm{mM}$ sodium/potassium phosphate, $\mathrm{pH} 7.0$, containing $0.02 \%$ sodium azide and $0.5 \mathrm{mM}$ dithiothreitol (buffer B). Proteins were further purified by gel filtration on a Superdex 75 column $(2 \times 60 \mathrm{~cm})$ which was developed with $360 \mathrm{~mL}$ of buffer B. The N-RS was eluted from $170 \sim 185 \mathrm{~mL}$ for N-RS whereas N-LumP was eluted from $210 \sim 220 \mathrm{~mL}$. Fractions were combined and concentrated by ultrafiltration (YM 10 membrane, Amicon). Protein concentration was determined by a modified Bradford method. ${ }^{21}$

Removal of ligand. Solutions containing $100 \mathrm{mM}$ phosphate, $\mathrm{pH} 7.0$, and the proteins N-RS and N-LumP, respectively, were dialyzed overnight against $100 \mathrm{mM}$ phosphate $\mathrm{pH}$ 7.0, containing $4 \mathrm{mM}$ dithiothreitol and $6 \mathrm{M}$ urea. For refolding, the apoprotein was dialyzed overnight against $100 \mathrm{mM}$ phosphate, pH 7.0 containing $0.3 \mathrm{mM}$ dithiothreitol and concentrated by ultrafiltration.

Fluorescence titration. Fluorescence experiments were performed on a Hitachi fluorescence spectrophotometer (FS-2000). Solution of 6,7-dimethyl-8-ribityllumazine in buffer A were added to solutions containing $5 \mu \mathrm{M}$ of proteins (N-RS or NLumP). Fluorescence was monitored by sequential addition of the fluorescent ligand (6,7-dimethyl-8-ribityllumazine: excitation, $410 \mathrm{~nm}$; emission, $490 \mathrm{~nm}$ ).

Cross-link experiment. Cross-link experiment was performed in $20 \mathrm{mM}$ HEPES buffer solution (10\% glycerol, $4 \mathrm{mM}$ DTT, $0.2 \mathrm{M} \mathrm{NaCl}$ ) incubating of $5 \mu \mathrm{M} \mathrm{N}-\mathrm{RS}$ or N-LumP by addition of glutaraldehyde to a concentration $0.05 \%(\mathrm{w} / \mathrm{v})$. The reaction mixture except cross linker of glutaraldehyde had been preincubated at room temperature for $10 \mathrm{~min}$. After addition of glutaraldehyde, the reaction was allowed to proceed for $1 \mathrm{~h}$, and stopped by $200 \mathrm{mM}$ Tris for $15 \mathrm{~min}$.

Acknowledgments. This work was supported by the exchanging program from Korea Research Foundation (614-2008-2C00004).

\section{References}

1. Hastings, J. W.; Nealson. K. H. Annu. Rev. Microbiol. 1977, 31, 549.

2. Meighen, E. A. Annu. Rev. Genet. 1994, $28,117$.

3. Koka, P.; Lee. J. Proc. Natl. Acad. Sci. USA 1979, 76, 3068.

4. O'Kane, D. J.; Lee. J. Biochemistry 1985, 12, 1467.

5. O'Kane, D. J.; Karle, V. A.; Lee. J. Biochemistry 1985, 24, 1461.

6. Beach, R. L.; Plaut, G. W. J. Am. Chem. Soc. 1970, 92, 2913.

7. Bacher, A.; Eberhardt, S.; Fischer, M.; Kis, K.; Richter, G. Annu. Rev. Nutr. 2000, 20, 153.

8. Lee, J. Biophys. Chem. 1993, 48, 149.

9. Petushkov, V. N.; Lee, J. Eur. J. Biochem. 1997, 240, 790.

10. O'Kane, D. J.; Woodward, B.; Lee, J.; Prasher. D. C. Proc. Natl. Acad. Sci. USA 1991, 88, 1100.

11. Lee, C. Y.; Meighen. E. A. J. Microbiol. 2000, 38, 80.

12. Chatwell, C.; Illarionov, V.; Ilaarionov, B.; Eisenreich, W.; Huber, R.; Skerra, A.; Bacher, A.; Fischer, M. J. Mol. Biol. 2008, 382, 44.

13. Truffault, V.; Coles, M.; Diercks, T.; Abelmann, K.; Eberhardt, S.; 
Lüttgen, H.; Bacher, A.; Kessler H. J. Mol. Biol. 2001, 309, 949.

14. Meining, W.; Eberhardt, S.; Bacher, A.; Ladenstein, R. J. Mol. Biol. 2003, 331, 1053.

15. Liao, D. I.; Wawrzak, Z.; Calabrese, J. C.; Viitanen, P. V.; Jordan, D. B. Structure 2001, 9, 399.

16. Illarionov, B.; Illarionova, V.; Lee, J.; van Dongen, W.; Vervoort, J. Biochem. Biophys. Acta 1994, 1201, 251.

17. Eberhardt, S.; Zingler, N.; Kemter, K.; Richter, G.; Gimbel, W.;
Cushman, M.; Bacher, A. Eur. J. Biochem. 2001, 268, 4315.

18. Eberhardt, S.; Richter, G.; Gimbel, W.; Werner, T.; Bacher, A. Eur. J. Biochem. 1996, 242, 712.

19. Illarionov, B.; Kempter, K.; Eberhardt, S.; Richter, G.; Cushman, M.; Bacher, A. J. Biol. Chem. 2001, 276, 11524.

20. Illarionov, B.; Eisenreich, W.; Wirth, M.; Lee, C. Y.; Woo, Y. E.; Bacher, A.; Fischer, M. Biol. Chem. 2007, 388, 1313.

21. Bradford, M. Anal. Biochem. 1976, 72, 248. 